\title{
Diseño de Ecuaciones de Sintonía para Controladores PID (Proporcional-Integral-Derivativo) Implementados en Fotobiorreactores
}

\author{
Arnaldo Verdeza, Leonardo Di Mare, Marco Sanjuán y Antonio Bula \\ Dpto. de Ingeniería Mecánica, Universidad del Norte, Barranquilla-Colombia \\ (e-mail: averdeza@uninorte.edu.co; Idimare@uninorte.edu.co; msanjuan@uninorte.edu.co; \\ abula@uninorte.edu.co)
}

Recibido Sep. 25, 2015; Aceptado Nov. 27, 2015; Versión final Ene. 29, 2016, Publicado Ago. 2016

\begin{abstract}
Resumen
Se desarrolló un conjunto de ecuaciones de sintonía para un fotobiorreactor usado para el cultivo de microalgas. El nivel de iluminación impacta significativamente en la tasa producción de biomasa y en los costos energéticos de este proceso, por lo que resulta deseable aprovechar la iluminación solar disponible. Sin embargo, la iluminación solar genera fluctuaciones en la energía incidente en el sistema que pueden conducir a oscilaciones en su temperatura, y afectar el comportamiento del sistema. Por lo tanto, se requiere emplear control automático para compensar estas perturbaciones y mantener las condiciones de operación. A partir de un modelo paramétrico de un fotobiorreactor, se desarrollaron las ecuaciones de sintonía para un controlador PID. Éste mantiene la temperatura del reactor mediante la manipulación del flujo de líquido de enfriamiento que pasa por el intercambiador de calor. Las ecuaciones de sintonía obtenidas mostraron un desempeño superior respecto a las ecuaciones $\lambda^{\prime}$ y mínimo error integral del valor absoluto (IAE) cuando se implementaron en un lazo de control por retroalimentación.
\end{abstract}

Palabras clave: control automático, fotobiorreactor, sintonía, PID

\section{Design of tuning equations for PID controllers (Proportional- Integral-Derivative) Implemented in Photo Bioreactors}

\begin{abstract}
A set of tuning equations specific for a photobioreactor used to produce biomass was developed. In this process, lighting levels impact significantly on biomass production rate and energy operation costs, being desirable to take advantage of available sun lighting. Nevertheless, the use of solar lighting leads to fluctuations in the incident energy into the system which, in turn, might cause oscillations in its temperature, affecting the system behavior. Therefore, automatic control is required to compensate the effect of these disturbances and maintain operating conditions. Hence, tuning equations were developed, from a parametric model of a photobioreactor, for PID controllers that maintain the reactor temperature at a set point by manipulating the coolant flow through the heat exchanger. The obtained tuning equations showed a better performance than $\lambda^{\prime}$ and minimum integral of absolute value of error (IAE) equations when implementing them in a feedback control loop in the photobioreactor model.
\end{abstract}

Keywords: automatic control, photobioreactor, tuning, PID 


\section{INTRODUCCIÓN}

El proceso de fotosíntesis es la fuente fundamental de casi toda la energía bioquímica del planeta y consiste en utilizar la energía de la luz solar para fabricar glúcidos y efectuar la biosíntesis de diversos compuestos orgánicos a partir de $\mathrm{CO}_{2}$ y $\mathrm{H}_{2} \mathrm{O}$, desprendiendo $\mathrm{O}_{2}$ al medio. Por tanto la posibilidad de utilizar los organismos fotosintéticos para la producción a gran escala de biomasa o productos energéticamente aprovechables resulta una alternativa factible para la generación de energías renovables. Así mismo, la utilización de microorganismos constituye una excelente opción para obtener productos de alto valor agregado o para realizar importantes transformaciones químicas de interés industrial (Rosello et al., 2007). Los organismos fotosintéticos como las microalgas habitan y prosperan en una amplia gama de condiciones ambientales, adaptándose especialmente a la disponibilidad de nutrientes y otras condiciones adversas e incluso hostiles. Esta versatilidad las convierte en una de las mejores opciones para ser utilizadas a nivel de producción comercial, porque garantiza una mejor adaptabilidad a las condiciones impuestas y ante diseños de equipos de cultivo robustos (Mata et al., 2010). Con el fin de aumentar la productividad y la escalabilidad de estos procesos biológicos, la atención se ha centrado en el diseño de equipos que puedan satisfacer las necesidades de los microorganismos fotosintéticos, pero aún existen muchos problemas de ingeniería sin resolver si se quieren desarrollar sistemas eficientes a bajo costo y de escalabilidad industrial (Barbosa et al., 2003; Janssen et al., 2003; Rosello et al., 2007). Hay dos alternativas principales para la producción de microorganismos fotosintetizadores: los sistemas abiertos en los que el cultivo está expuesto a la atmósfera y los sistemas cerrados, comúnmente denominados fotobiorreactores, en los que el cultivo tiene poco 0 ningún contacto con la atmósfera (Contreras et al., 2003).

La mayoría de los sistemas de producción industrial de biomasa de microalgas construidos antes de los años 90 fueron esencialmente sistemas abiertos tipo carrusel, que permiten alcanzar densidades celulares de hasta $0,7 \mathrm{~g}$ de células (base seca) por litro. Estos sistemas, constituidos por canales poco profundos (nivel de agua entre 15-20 $\mathrm{cm}$ ) en forma de circuito cerrado en los que el medio de cultivo es impulsado mediante paletas rotatorias, generalmente requieren de grandes áreas de terreno (500-5000 $\left.\mathrm{m}^{2}\right)$, elevados consumos de agua para compensar las pérdidas por evaporación y están sujetas a la afectación por las lluvias, el viento y las especies animales, aunque tienen como ventaja el bajo costo de producción de biomasa en algunas zonas geográficas específicas. La baja densidad celular origina también varios inconvenientes, que incluyen la baja productividad, la costosa recuperación del producto de medios diluidos y la dificultad para el control de la temperatura, por lo que las especies más aptas para crecer en los reactores son las adaptadas a las condiciones más extremas (como Spirullina, Dunaliella y Chlorella), lo que origina otra dificultad pues la disposición de las aguas residuales debe ser más exigente para prevenir la contaminación biológica por dichas especies (Pramparo et al., 2008). Estos inconvenientes estimularon el desarrollo de fotobiorreactores, equipos confinados construidos con materiales transparentes como vidrio y policarbonato, entre otros materiales (Sanchez et al., 2002), (Sierra et al., 2008); incluso bolsas plásticas suspendidas en parrillas flotantes en lagunas o en el océano (Richmond et al., 2004). Los fotobiorreactores son una de las mejores alternativas para este tipo de procesos debido a que están diseñados para reproducir microorganismos fotosintéticos y operan suministrando los requerimientos óptimos de luz, temperatura, pH, agitación y transferencia de masa (disponibilidad de nutrientes) (Pulz et al., 2001). En este tipo de cultivos se requiere de un control estricto de estos parámetros para mantener las condiciones ideales de crecimiento y de este modo garantizar una mayor productividad, un mejor aprovechamiento de los insumos utilizados y la manipulación de los comportamientos metabólicos de estos organismos (Chisti et al., 1988).
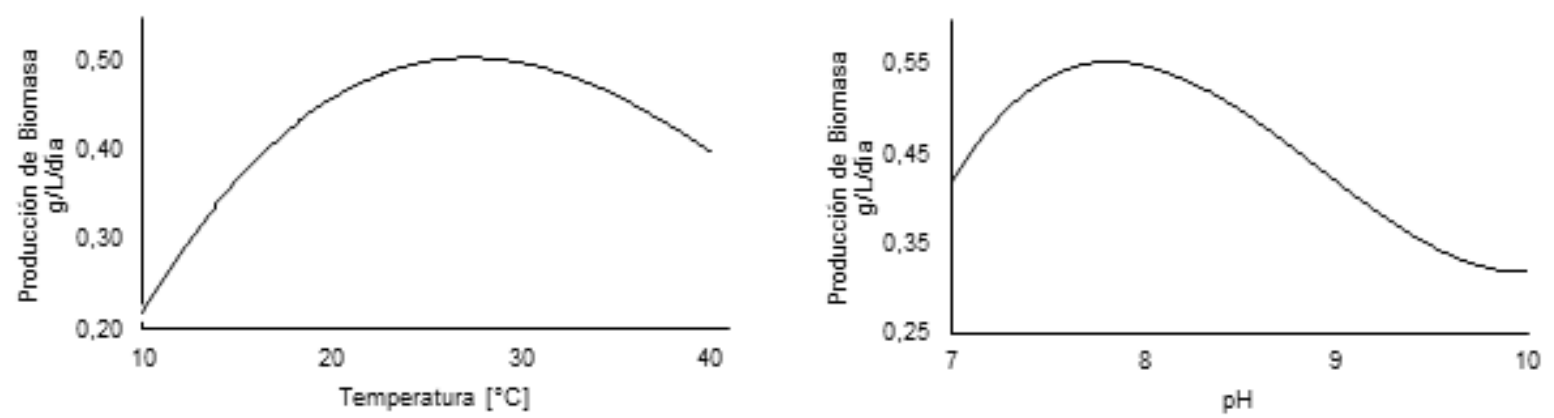

Fig. 1. Dependencia de la temperatura y el pH, Scenedesmus almeriensis. (Sánchez et al., 2008)

Dentro de los parámetros de cultivo de microalgas la temperatura es uno de los más importantes, como se puede apreciar en la Fig. 1., y debe ser cuidadosamente controlada teniendo en cuenta que las condiciones pueden variar mucho dentro de una instalación de cultivo o de laboratorio (Andersen et al., 2005). Los 
sistemas fotosintéticos generan calor debido a la ineficiencia de la fotosíntesis para convertir la energía luminosa en energía química (Morita et al., 2001; Pirt, 1983), lo cual se evidencia si se considera que la irradiación absorbida por los compuestos fotosintéticos en las plantas para su conversión en energía química no supera el $16 \%$ de la radiación total que recibe, y un cultivo denso de microorganismos fotosintéticos en agua con $10 \mathrm{~cm}$ de profundidad absorbe menos del 38\%, como se puede apreciar en el artículo "Spectral Signatures of Photosynthesis. I. Review of Earth Organisms" (Kiang et al., 2007)

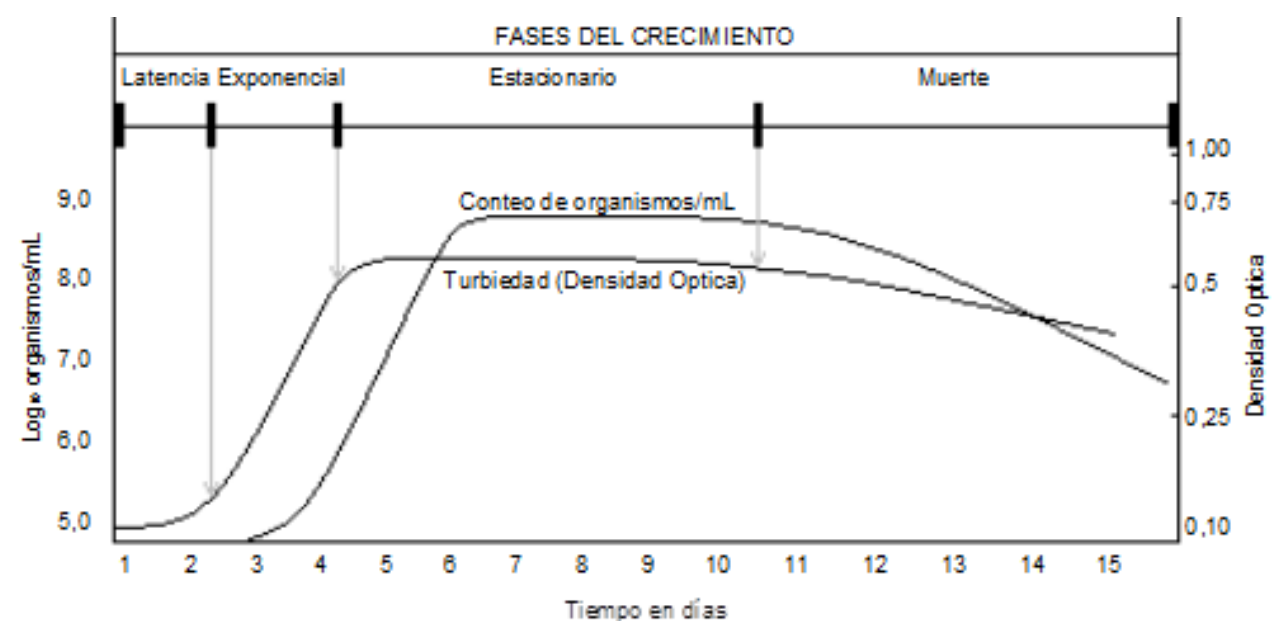

Fig. 2. Curva de crecimiento típica de microorganismos fotosintéticos.

Ahora, si se observa una curva de crecimiento típico, como la mostrada en la Fig. 2., es de notarse que para la curva de opacidad la mayor tasa de cambio en la curva va del el día 2 al 5, esto suma un total de aproximadamente 72 horas en las que la opacidad del cultivo pasa de 0,15 a 0,5 puntos y que la energía máxima contenida en la radiación que llega a la superficie terrestre no excede los $1200 \mathrm{~W} / \mathrm{m}^{2}$. La variación del calor absorbido por causa del aumento en la densidad del cultivo en el sistema estaría alrededor de los $7 \mathrm{~W} / \mathrm{m}^{2} / \mathrm{h}$, mientras que la variación impuesta por la radiación solar que estaría entre los $80 \mathrm{~W} / \mathrm{m}^{2} / \mathrm{h}$. La conversión teórica de la luz roja en energía química (NADPH) es sólo del $31 \%$, mientras que el $69 \%$ se pierde como calor. Así entonces, la cantidad de refrigeración requerida depende de la intensidad de la luz incidente y la concentración de células (es decir, cantidad de luz que se absorbe), por tanto la refrigeración en el proceso de cultivo es necesaria. La temperatura afecta en mayor o menor medida a las microalgas dependiendo de la especie. Principalmente afecta factores como la tasa de crecimiento (Lee, 1988), (Yokoya, 1992), (Dauta, 1990), y las concentraciones y tipos de compuestos metabólicos que éstas producen (Chena, 2008), (Converti, 2009). Por tanto, el grado de control de este parámetro influirá en significativamente sobre la productividad y eficiencia del cultivo de microalgas, así como en la calidad y cantidad de los subproductos que se obtengan de éste.

\section{MODELAMIENTO DEL FOTOBIORREACTOR}

El reactor, de $10 \mathrm{~cm}$ de profundidad y $1 \mathrm{~m}^{2}$ de superficie, se muestra en la Fig. 3.

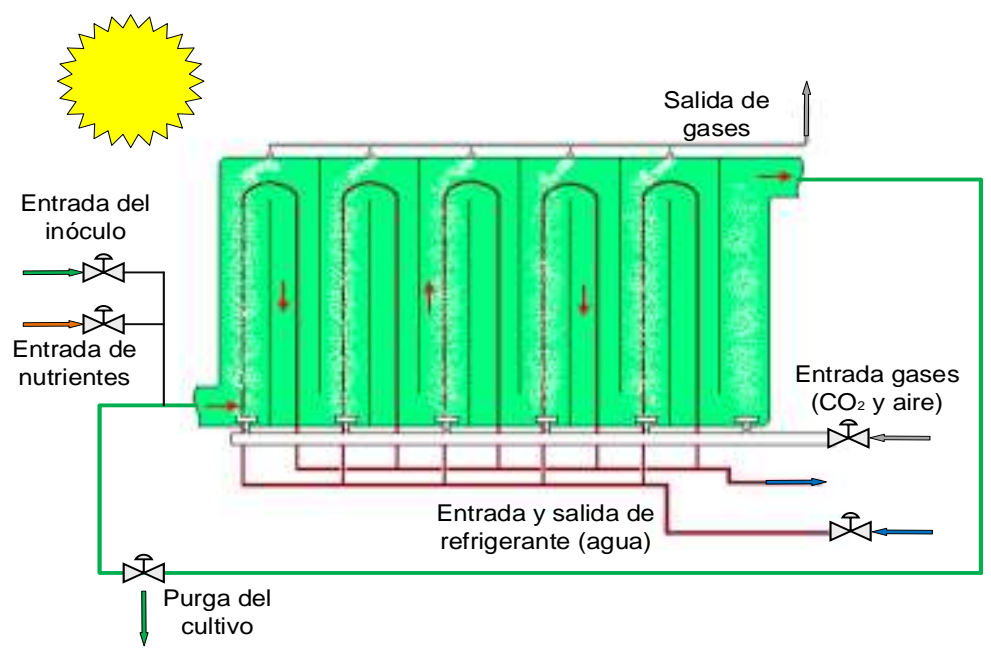

Fig. 3. Esquemático de la situación considerada 
El sistema se encuentra expuesto a la radiación solar durante el día, entre las 6:00 am y 6:00 pm, mientras que, durante la noche, entre las 6:00 pm y las 6:00 am, se mantiene con iluminación artificial. La luz que incide sobre el sistema suministra una cantidad de energía más que suficiente para que los microorganismos desarrollen sus procesos fotosintéticos. Dado que las microalgas alcanzan sus mayores tasas de crecimiento a una temperatura próxima a los $26^{\circ} \mathrm{C}$, es deseable remover el exceso de energía suministrado y mantener constante la temperatura. A continuación se ilustra el balance de energía del proceso. Las ecuaciones enunciadas emplean la nomenclatura mostrada en la Tabla 1.

$\dot{q}_{l u z}+\dot{q}_{a l}+\dot{q}_{m e d}-\dot{q}_{a g}-\dot{q}_{a i r}-\dot{q}_{a m b}=\frac{d E_{r e a c}}{d t}$

Para los efectos del análisis térmico se considerará como una perturbación el cambio en el nivel de irradiación lumínica y se considera que el metabolismo de las microalgas es constante. La variable de salida del modelo será la temperatura del estanque; con ella se determinará el ajuste para la válvula que regula el flujo de agua (refrigerante) a través del serpentín de enfriamiento, como se ilustra en Fig. 4.

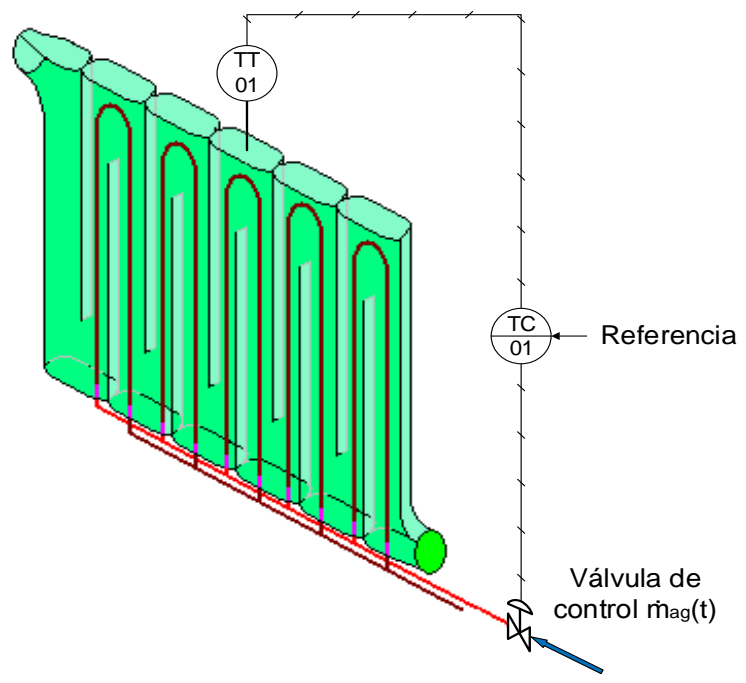

Fig. 4. Esquema de control

El balance de masa queda:

$\dot{q}_{l u z}+\dot{q}_{a l}+\dot{q}_{\text {med }}-\dot{q}_{a g}-\dot{q}_{a i r}-\dot{q}_{a m b}=\frac{d\left(m_{e s t} C_{p} \Delta T_{\text {reac }}\right)}{d t}$

Donde:

$\dot{q}_{a g}=\dot{m}_{a g} C_{p} \Delta T_{a g}$

$\Delta T_{a g}=T_{o}-T_{i}$

$m_{e s t}=V \rho$

Remplazando las ecuaciones (3), (4) y (5) en la ecuación (1) resulta:

$\dot{q}_{l u z}+\dot{q}_{a l}+\dot{q}_{m e d}-\dot{m}_{a g} C_{p}\left(T_{o}-T_{i}\right)-\dot{q}_{a i r}-\dot{q}_{a m b}=V \rho C_{p} \frac{d\left(\Delta T_{\text {reac }}\right)}{d t}$

Como el tercer término de la izquierda no es lineal, se lleva a cabo una linealización por series de Taylor para poder aplicar la transformada de Laplace:

$$
\dot{m}_{a g} C_{p}\left(T_{o}-T_{i}\right)=\dot{m}_{a g 0} C_{p}\left(T_{o}-T_{i}\right)_{0}+C_{p}\left(T_{o}-T_{i}\right)_{s s}\left(\dot{m}_{a g}-\dot{m}_{a g 0}\right)+\dot{m}_{a g 0} C_{p}\left(T_{o}-T_{o 0}\right)-\dot{m}_{a g 0} C_{p}\left(T_{i}-T_{i 0}\right)
$$


$\mathrm{Si}$, como se espera, que suministro del agua de enfriamiento se derive de un sistema que proporcione una temperatura constante; se puede eliminar el último término, ya que $T_{i}=T_{i 0}=c t e$, por tanto se tiene:

$\dot{m}_{a g} C_{p}\left(T_{o}-T_{i}\right)=\dot{m}_{a g 0} C_{p}\left(T_{o}-T_{i}\right)_{0}+C_{p}\left(T_{o}-T_{i}\right)_{s s}\left(\dot{m}_{a g}-\dot{m}_{a g 0}\right)+\dot{m}_{a g 0} C_{p}\left(T_{o}-T_{o 0}\right)$

Una vez linealizado este término, se sustituye en la ecuación (6) para derivar la ecuación dinámica que describe la temperatura en el fotobiorreactor:

$$
\begin{aligned}
\dot{q}_{l u z}+\dot{q}_{a l}+\dot{q}_{\text {med }}-\dot{m}_{a g 0} C_{p}\left(T_{o}-T_{i}\right)_{0}-C_{p}\left(T_{o}-T_{i}\right)_{s s}\left(\dot{m}_{a g}-\dot{m}_{a g 0}\right)-\dot{m}_{a g 0} C_{p}\left(T_{o}-T_{o 0}\right) & \\
& -\dot{q}_{a i r}-\dot{q}_{a m b}=V \rho C_{p} \frac{d\left(\Delta T_{\text {reac }}\right)}{d t}
\end{aligned}
$$

También se obtiene la ecuación en estado estable:

$\dot{q}_{l u z 0}-\dot{q}_{a l g 0}-\dot{m}_{a g 0} C_{p}\left(T_{o}-T_{i}\right)_{0}-\dot{q}_{a i r 0}=0$

Para trabajar con variaciones, se calcula la diferencia entre la ecuación dinámica y la de estado estable:

$$
\begin{array}{r}
\dot{q}_{l u z}+\dot{q}_{a l}+\dot{q}_{\text {med }}-\dot{m}_{a g 0} C_{p}\left(T_{o}-T_{i}\right)_{0}-C_{p}\left(T_{o}-T_{i}\right)_{s s}\left(\dot{m}_{a g}-\dot{m}_{a g 0}\right)-\dot{m}_{a g 0} C_{p}\left(T_{o}-T_{o 0}\right)-\dot{q}_{a i r}-\dot{q}_{a m b} \\
-\left(\dot{q}_{l u z 0}+\dot{q}_{a l g 0}-\dot{m}_{a g 0} C_{p}\left(T_{o}-T_{i}\right)_{0}-\dot{q}_{a i r 0}\right)=V \rho C_{p} \frac{d\left(\Delta T_{r e a c}\right)}{d t}
\end{array}
$$

Considerando que la variación en el ingreso de calor por la radiación es mucho más rápida que la absorbida por los microorganismos a medida que se densifica el cultivo, se considera que la variación del calor absorbido por el metabolismo de las algas se mantendrá constante por lo que el calor asociado a él no experimentará una variación significativa para efectos del control de la temperatura del sistema. Por otra parte la permanencia de los gases inyectados al sistema no es suficiente para propiciar una extracción de calor significativa, al igual que las pérdidas de calor al medio circundante y la entrada por suministro de medio nutrido. Se tiene entonces la siguiente expresión en variables de desviación:

$\digamma_{\dot{q}_{l u z}}(t)-C_{p}\left(T_{o}-T_{i}\right)_{s S} \digamma_{\dot{m}_{a g}}(t)-\dot{m}_{a g 0} C_{p} \digamma_{T_{o}}(t)=V \rho C_{p} \frac{d\left(\Delta T_{\text {reac }}\right)}{d t}$

Como una simplificación adicional, se considera que la temperatura de salida del agua es directamente proporcional a la temperatura del estanque: $T_{o}=\mu T_{\text {reac }}$. De forma que la ecuación anterior quedaría de la siguiente forma:

$\Gamma_{\dot{q}_{l u z}}(t)-C_{p}\left(T_{o}-T_{i}\right)_{s S} \digamma_{\dot{m}_{a g}}(t)-\dot{m}_{a g 0} C_{p} \mu \Gamma_{T_{\text {reac }}}(t)=V \rho C_{p} \frac{d\left(\Delta T_{\text {reac }}\right)}{d t}$

Al llevar a cabo la transformada de Laplace se obtiene la siguiente expresión:

$\dot{Q}_{\text {luz }}(s)-C_{p}\left(T_{o}-T_{i}\right)_{s s} \dot{M}_{a g}(s)-\dot{m}_{a g 0} C_{p} \mu T_{\text {reac }}(s)=V \rho C_{p} s T_{\text {reac }}(s)$

Simplificando:

$\frac{1}{\dot{m}_{a g 0} C_{p} \mu} \dot{Q}_{l u z}(s)-\frac{C_{p}\left(T_{o}-T_{i}\right)_{s s}}{\dot{m}_{a g 0} C_{p} \mu} \dot{M}_{a g}(s)=\left(\frac{V \rho C_{p}}{\dot{m}_{a g 0} C_{p} \mu} s+1\right) T_{\text {reac }}(s)$

Lo anterior resulta equivalente a una función de primer orden:

$T_{\text {reac }}(s)=\frac{K_{1}}{(\tau s+1)} \dot{Q}_{l u z}(s)-\frac{K_{2}}{(\tau s+1)} \dot{M}_{a g}(s)$

Donde:

$\tau=\frac{V \rho}{\mu \dot{m}_{a g 0}}$ 
$K_{1}=\frac{1}{\dot{m}_{a g 0} C_{p} \mu}$

$K_{2}=\frac{\left(T_{o}-T_{i}\right)_{s s}}{\dot{m}_{a g 0} \mu}$

Las funciones de transferencia se determinan de forma directa:

$$
\begin{gathered}
G_{1}(s)=\frac{T_{r e a c}(s)}{\dot{Q}_{l u z}(s)}=\frac{K_{1}}{(\tau s+1)} \\
G_{2}(s)=\frac{T_{r e a c}(s)}{\dot{M}_{a g}(s)}=\frac{-K_{2}}{(\tau s+1)}
\end{gathered}
$$

Con esta información se puede construir el diagrama de bloques del proceso y, con él, modelar el sistema en Simulink. En la Fig. 5 se presenta el diagrama de bloques correspondiente al lazo cerrado de control por retroalimentación.

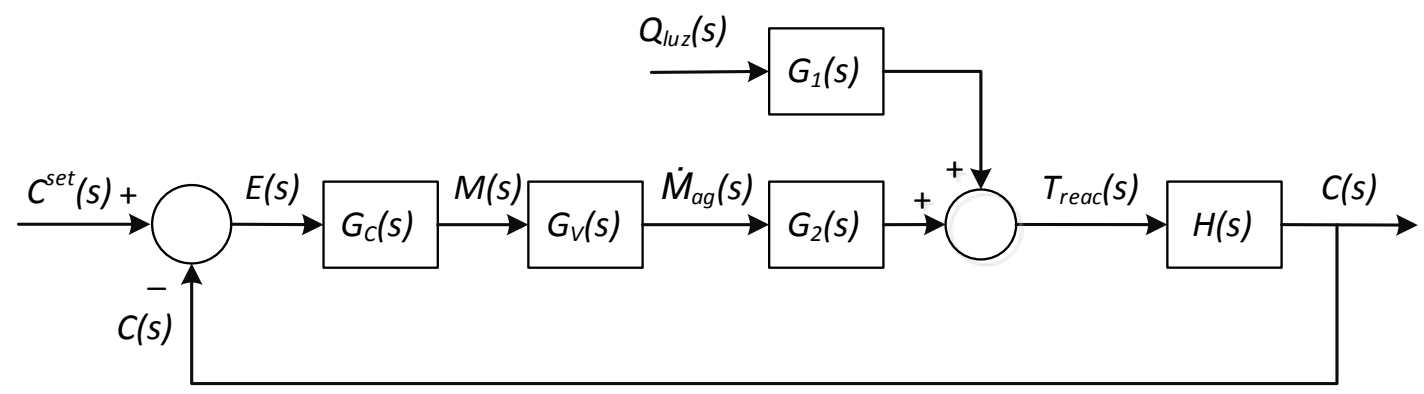

Fig. 5. Función de transferencia del proceso (en el lazo cerrado de control).

Donde $C^{\text {set }}(s)$ es el "setpoint" o referencia de temperatura, $C(s)$ es la señal del sensor/transmisor, $E(s)$ es el error, $G_{c}(s)$ es la función de transferencia del controlador, $G_{v}(s)$ es la función de transferencia de la válvula de control, $M(s)$ es la señal de salida del controlador y $H(s)$ es la función de transferencia del sensor/transmisor. Con el fin de obtener expresiones para los parámetros de la función de transferencia $G_{2}(s)$ en función de parámetros del reactor que sean fácilmente modificables, se consideró que el calor ganado $\left(\dot{q}_{l u z}\right)$ es igual al que absorbe el agua, de forma que:

$\dot{q}_{l u z 0}=\dot{m}_{a g 0} C_{p}\left(T_{o}-T_{i}\right)_{s s}$

$\rightarrow K_{2}=\frac{\dot{q}_{l u z 0}}{\dot{m}^{2}{ }_{a g 0} C_{p} \mu}$

Definiendo:

$\dot{q}_{A l u z 0}=\frac{\dot{q}_{l u z 0}}{A}=\frac{\dot{q}_{l u z 0}}{V / h}$

Por tanto, queda:

$K_{2}=\frac{\mu \tau^{2} \dot{q}_{\text {Aluz } 0}}{h V \rho^{2} C_{p}}$

Si se considera que el medio para el proceso será siempre agua dulce (densidad y calor específico constantes) y que la altura ideal para los fotobiorreactores depende de la distancia óptima que debe recorrer el rayo de luz para el crecimiento de las algas, se pueden agrupar estos términos en una constante y se obtiene que:

$K_{2}=\frac{\varepsilon \mu \tau^{2} \dot{q}_{\text {Asolo }}}{V}$ 
Donde:

$\varepsilon=\frac{1}{h \rho^{2} C_{p}}$

Una vez encontradas las expresiones para los parámetros de $G_{2}(s)$, se define:

$G(s)=G_{v}(s) G_{2}(s) H(s)$

Aquí:

$G_{v}(s)=\frac{K_{v}}{\tau_{v} s+1}$

Es la función de transferencia de la válvula de control, y $H(s)=K_{T}$ es la función de transferencia del sensor/transmisor de temperatura. Si se considera que la respuesta de la apertura de la válvula de control es significativamente más rápida que la de la temperatura del reactor, se puede escribir:

$G(s)=\frac{-K_{v} K_{2} K_{T}}{(\tau s+1)}=\frac{-K_{2}{ }^{\prime}}{(\tau s+1)}$

Donde:

$K_{2}^{\prime}:=K_{v} K_{2} K_{T}$

\section{DESARROLLO DE ECUACIONES DE SINTONÍA}

Inicialmente se realiza un muestreo con las posibles condiciones que pueden tomar las diferentes variables del proceso para determinar, con las relaciones (26) y (31), los valores máximos y mínimos de $K_{2}{ }^{\prime}$. En la condición en que la ganancia es máxima, el valor encontrado de $K_{2}{ }^{\prime}$ se toma como divisor de los demás para así estandarizar las variables de experimentación. Los valores de $\tau$ se seleccionan con base en las limitaciones del proceso, considerándose el flujo de agua necesario para retirar el calor suministrado por radiación y el volumen del reactor, dada una eficiencia, tal que se alcance a retirar el calor en la condición más crítica. Para el tiempo muerto, se considera el rango entre el $0,2 \tau$ y $0,5 \tau$, valores acordes con la dinámica del proceso en condiciones normales de funcionamiento, de forma que se define una variable experimental como la razón $t_{0} / \tau$, asociada a las variables consideradas para la selección de los rangos de $K_{2}{ }^{\prime}$ y $\tau$. Con estas se plantea un diseño multifactorial $3^{3}$ con el fin de realizar un análisis completo del comportamiento de las ecuaciones de sintonía a obtener.

Tabla 1. Factores para el diseño experimental.

\begin{tabular}{|c|c|c|c|}
\hline Factor/Nivel & $K_{2}{ }^{\prime}$ & $\tau(\mathrm{h})$ & $t_{0} / \tau$ \\
\hline Bajo [-1] & 0,1 & 0,50 & 0,20 \\
\hline Medio [ 0 ] & 0,3 & 1.00 & 0,35 \\
\hline Alto [ 1 ] & 0,5 & 1,50 & 0,50 \\
\hline
\end{tabular}

Dado que el crecimiento de las algas resulta sensible a las variaciones en la temperatura y que la oscilación en la señal que el controlador envía a la válvula genera desgaste prematuro en la misma, para el diseño de las ecuaciones de sintonía se tomaron como parámetros a minimizar; $\int|e(t)| d t$ que corresponde a la integral del valor absoluto del error (IAE), $\sigma^{2}(C(t))$ la oscilación de la señal del sensor/transmisor y $\sigma^{2}(M(t))$ la oscilación en la señal del controlador a la válvula (varianzas de ambos parámetros).

$f_{\text {optm }}\left(K_{c}, \tau_{I}, \tau_{D}\right)=\int|e(t)| d t+\sigma^{2}(C(t))+\sigma^{2}(M(t))$

Con la función objetivo (33), se empleó la función fmincon de MatLab® por su capacidad para encentrar el mínimo de una función no lineal de varias variables incorporando restricciones (MathWorks, 2015). Esto permite obtener los mejores parámetros de sintonía $K_{C}, \tau_{I}$ y $\tau_{D}$ para cada condición simulada, dados $K_{2}{ }^{\prime}, \tau$ y $t_{0} / \tau$. Además se especifican los límites de la simulación para cada uno de los parámetros a determinar, de forma que excedieran los valores extremos arrojados por las técnicas tradicionales de sintonía, $\lambda^{\prime}$ y mínimo IAE, los cuales se muestran en la Tabla 2.

El método de sintonía $\lambda^{\prime}$ está diseñado para obtener una respuesta específica mediante el ajuste de un solo parámetro, lo cual permite controlar el nivel de amortiguamiento en la variable de salida, ideal para para procesos donde el tiempo muerto y la constante de tiempo del proceso son relevantes respecto a la 
ganancia, en otras palabras es ideal para ajustar la sintonía del controlador a procesos lentos. Por otro lado el criterio de sintonía mínimo IAE da como resultado una ganancia para el controlador más alta y respuestas aunque más rápidas, más oscilatorias (Smith C., et al., 2006). La Tabla 3, muestra las correlaciones utilizadas para obtener los parámetros de sintonía $\left(K_{C}, \tau_{I}\right.$ y $\left.\tau_{D}\right)$ del controlador. Nótese que las ecuaciones utilizadas son para cambios en la referencia de la variable controlada o "setpoint" dado precisamente que este tipo de ajuste reduce las oscilaciones en la respuesta.

Tabla 2. Selección de los límites de optimización.

\begin{tabular}{|c|c|c|c|c|c|c|}
\hline & \multicolumn{2}{|c|}{$\lambda^{\prime}$} & \multicolumn{2}{c|}{ Mínimo IAE } & \multicolumn{2}{c|}{ Empleado } \\
\cline { 2 - 7 } & Máx & Mín & Máx & Mín & Máx & Mín \\
\hline$K_{C}$ & 22,727 & 2,7 & 60 & 6,86 & 100 & 0,1 \\
\hline$\tau_{I}$ & 1,5 & 0,5 & 1,5 & 0,2 & 20 & 0,01 \\
\hline$\tau_{D}$ & 0,263 & 0,05 & 0,263 & 0,05 & 5 & 0,01 \\
\hline
\end{tabular}

Tabla 3. Ecuaciones de sintonía mínimo IAE y $\lambda^{\prime}$.

\begin{tabular}{|c|c|c|}
\hline \multirow{2}{*}{ Ecuaciones de sintonía mínimo IAE } & \multicolumn{2}{|c|}{ Coeficientes } \\
\cline { 2 - 3 }$K_{c}=\frac{a}{K_{2}^{\prime}}\left(\frac{t_{0}}{\tau}\right)^{b}$ & 1,086 & $-0,869$ \\
\hline$\tau_{I}=\frac{\tau}{a+b\left(\frac{t_{0}}{\tau}\right)}$ & 0,740 & -0130 \\
\hline$\tau_{D}=a \tau\left(\frac{t_{0}}{\tau}\right)^{b}$ & 0,348 & 0,914 \\
\hline Ecuaciones de sintonía $\lambda^{\prime}$ & \multirow{2}{*}{ Parámetro de ajuste } \\
\hline$K_{c}=\frac{\tau}{K_{2}^{\prime}\left(\lambda^{\prime}+t_{0}\right)}$ & \multirow{2}{*}{} \\
\hline$\tau_{I}=\tau$ & \multicolumn{2}{|c}{$\lambda^{\prime}=1,2 t_{0}$} \\
\hline$\tau_{D}=\frac{t_{0}}{2}$ & \multicolumn{2}{|c}{} \\
\hline
\end{tabular}

\section{RESULTADOS}

En esta sección se presentan los resultados del diseño experimento factorial realizado, así como las ecuaciones de sintonía derivadas de dichos resultados mediante regresiones. Además, se hace una evaluación del desempeño de las ecuaciones de sintonía obtenidas, comparándolas con las ecuaciones $\lambda^{\prime}$ y Mínimo IAE.

\section{Ecuaciones de Sintonía}

Los resultados arrojados por la simulación se adjuntan en la Tabla 4. A partir de los cuales se desarrollaron distintos modelos de regresión. El modelo con mejor ajuste se muestra en la Tabla 5 . Nótese que el $\tau_{D}$ se fija en 0 , ya que en la mayoría de las simulaciones se observó que el algoritmo de optimización le asignaba a este parámetro el límite inferior del intervalo de simulación. Esto se debe a que la función objetivo a minimizar incluye las varianzas de las señales del sensor y el controlador, y el tiempo derivativo induce inestabilidad al lazo de control.

\section{Desempeño de las Ecuaciones de Sintonía}

A continuación se lleva a cabo una comparación cualitativa y cuantitativa del desempeño de las ecuaciones de sintonía obtenidas en este artículo con las ecuaciones $\lambda^{\prime}$ y mínimo IAE. Los criterios de evaluación cualitativa serán el tiempo de estabilización y el número de oscilaciones de la respuesta, mientras que los criterios cuantitativos serán la integral del valor absoluto del error (IAE) y las varianzas de las señales $m(t)$ y $C(t)$ respectivamente. Se han identificado dos condiciones de operación particularmente difíciles de controlar en el fotobiorreactor, las cuales se presentan cuando $K_{2}^{\prime}=0.5, \tau=0.5$ y $t_{0} / \tau=0.2$, y cuando $K_{2}^{\prime}=$ $0.5, \tau=1.5$ y $t_{0} / \tau=0.5$ respectivamente. Por lo tanto, se utilizarán estos valores para llevar a cabo dos escenarios de evaluación de desempeño. 


\section{Escenario de Evaluación 1}

$K_{2}^{\prime}=0.5, \tau=0.5$ y $t_{0} / \tau=0.2$; Como se desea evaluar la respuesta del lazo de control tanto para cambios de referencia (setpoint) como para perturbaciones, se hace un cambio de referencia de $50 \%$ TO a 55\%TO en $t=1$ minutos, y luego de se hace un cambio en la tasa de suministro de calor por radiación, $\dot{q}_{l u z}$, que es la principal perturbación. Dicho cambio representa un $50 \%$ del cambio en la referencia $\left(C^{s e t}(s)\right)$, en $t=10$ minutos. En la Fig. 6 se muestra la respuesta de la variable controlada $(C(t))$ para cada uno de los sets de sintonía a evaluar ante el cambio de referencia y la perturbación inducidos, la Fig 7 contiene la respuesta del controlador o señal enviada a la válvula.

Tabla 4. Resultados de la evaluación experimental.

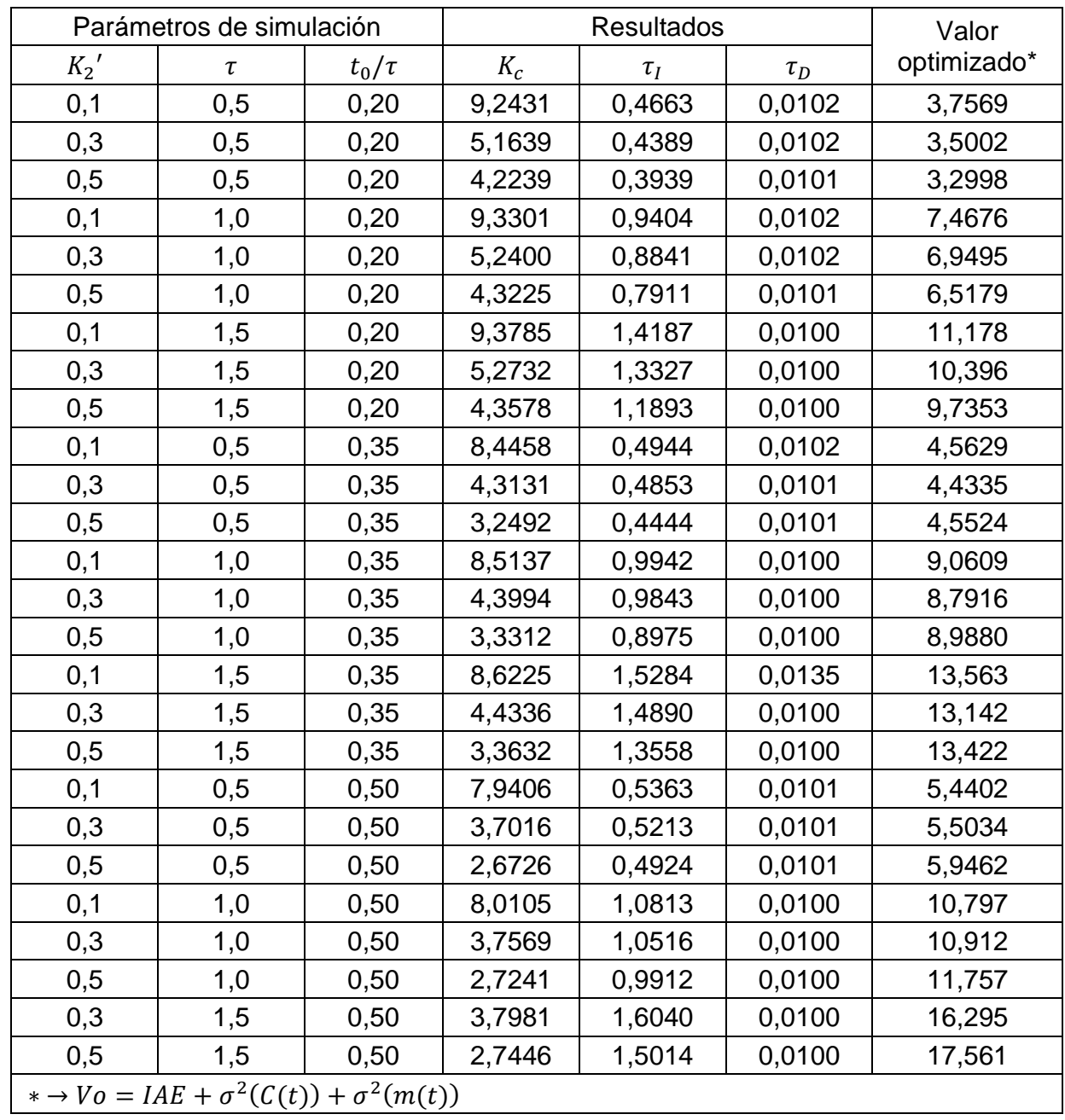

Tabla 5. Ecuaciones de sintonía y coeficientes encontrados.

\begin{tabular}{|c|c|c|c|c|}
\hline \multirow{2}{*}{ Ecuación } & \multicolumn{3}{|c|}{ Coeficientes } & \multirow{2}{*}{$R^{2}$} \\
\cline { 2 - 4 }$K_{c}=\frac{a}{K_{2}{ }^{b}}\left(\frac{\tau}{t_{0}}\right)^{-c}$ & 1,7449 & 0,5772 & 0,2340 & 0,9847 \\
\hline$\tau_{I}=a \tau\left(\frac{t_{0}}{\tau}\right)^{b}$ & 1,2147 & 0,2078 & - & 0,9801 \\
\hline$\tau_{D}=0$ & - & - & - & - \\
\hline
\end{tabular}

Es notable que la respuesta obtenida con las ecuaciones de sintonía propuestas presenta un menor tiempo de estabilización ante el cambio de referencia con respecto a la respuesta obtenida con sintonía mínimo IAE, mientras que un tiempo de respuesta similar al obtenido con $\lambda^{\prime}$, presentando además menos oscilaciones. En cuanto a los parámetros cuantitativos de desempeño, se obtuvieron también mejores resultados con las ecuaciones propuestas, tal como se evidencia en la Tabla 7, donde se resumen los resultados obtenidos. 


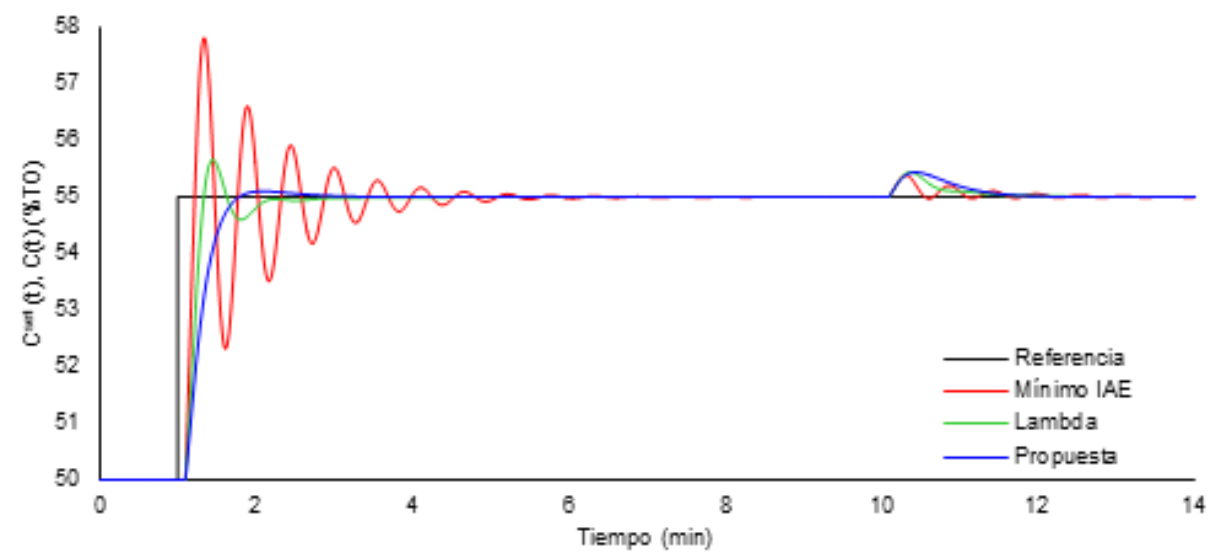

Fig. 6. Respuesta de la variable controlada $(C(t))$ para cada uno de los sets de sintonía, escenario de evaluación $1: K_{2}^{\prime}=0.5, \tau=0.5$ y $t_{0} / \tau=0.2$.

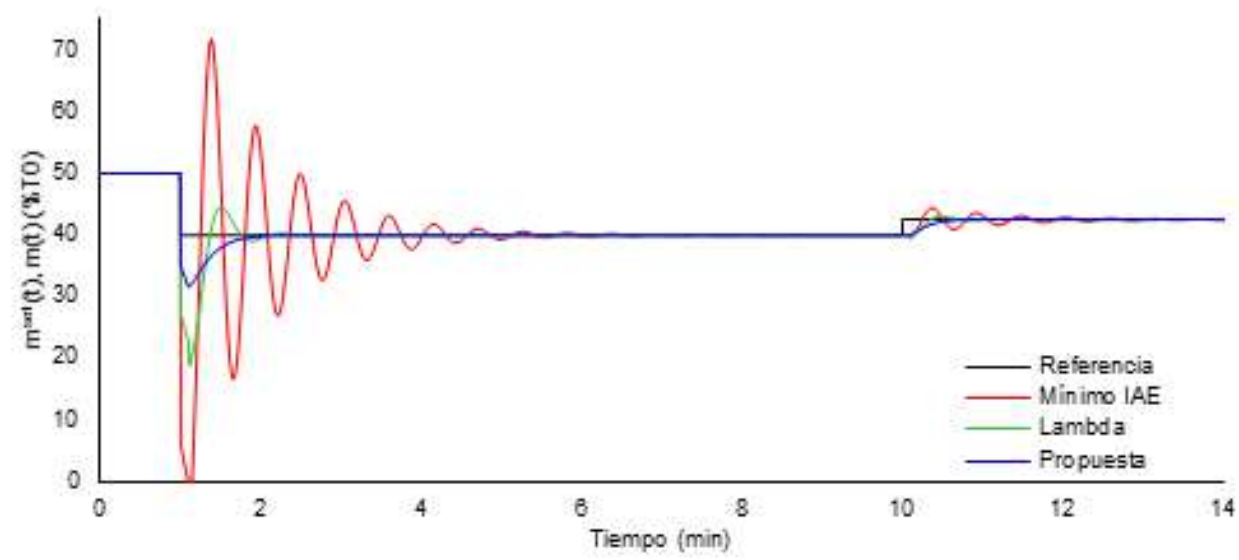

Fig. 7. Respuesta del controlador $(m(t))$ para cada uno de los sets de sintonía, escenario de evaluación 1: $K_{2}^{\prime}=0.5, \tau=0.5$ y $t_{0} / \tau=0.2$.

Tabla 6. Parámetros cuantitativos de desempeño Integral absoluta del error (IAE) la varianza de la señal del transmisor y salida del controlador, para el escenario de evaluación 1.

\begin{tabular}{|c|c|c|c|c|}
\hline Ecuaciones de Sintonía & IAE & $\sigma^{2}(C(t))$ & $\sigma^{2}(m(t))$ & $V o$ \\
\hline Mínimo IAE & 3,211 & 0,328 & 29,248 & 32,787 \\
\hline$\lambda^{\prime}$ & 1,622 & 0,225 & 3,899 & 5,746 \\
\hline Propuesta & 1,979 & 0,281 & 0,902 & 3,162 \\
\hline
\end{tabular}

\section{Escenario de Evaluación 2}

$K_{2}^{\prime}=0.5, \tau=1.5$ y $t_{0} / \tau=0.5$; Para llevar a cabo la evaluación en este escenario se induce el mismo cambio de referencia y la misma perturbación hechos en el escenario 1, aunque en esta ocasión la perturbación se hace en $t=30$ minutos. En la Fig. 9 se muestran las respuestas obtenidas y la Fig 10 contiene la señal enviada a la válvula por el controlador. Al igual que en el escenario 1, en este escenario las ecuaciones de sintonía propuestas mostraron una respuesta con menos tiempo de estabilización y menos oscilaciones, así como también un mejor desempeño en cuanto a los criterios cuantitativos de evaluación, tal como se evidencia en la Tabla 8, donde se resumen los resultados obtenidos.

\section{CONCLUSIONES}

Se desarrollaron ecuaciones de sintonía para mejorar el desempeño del lazo de control de temperatura de un fotobioreactor usado para el cultivo de microalgas. Estas ecuaciones fueron desarrolladas a partir de un modelo paramétrico del fotobioreactor y el algoritmo de optimización fmincon de Matlab®. Se llevó a cabo un experimento factorial $3^{3}$ con los parámetros del modelo, y en cada condición experimental la función objetivo a minimizar es la suma del IAE y las varianzas de las señales del transmisor y el controlador respectivamente, ya que estas tres variables son críticas para la operación del reactor. A partir de los datos se obtuvieron, mediante regresiones, las ecuaciones de sintonía. 


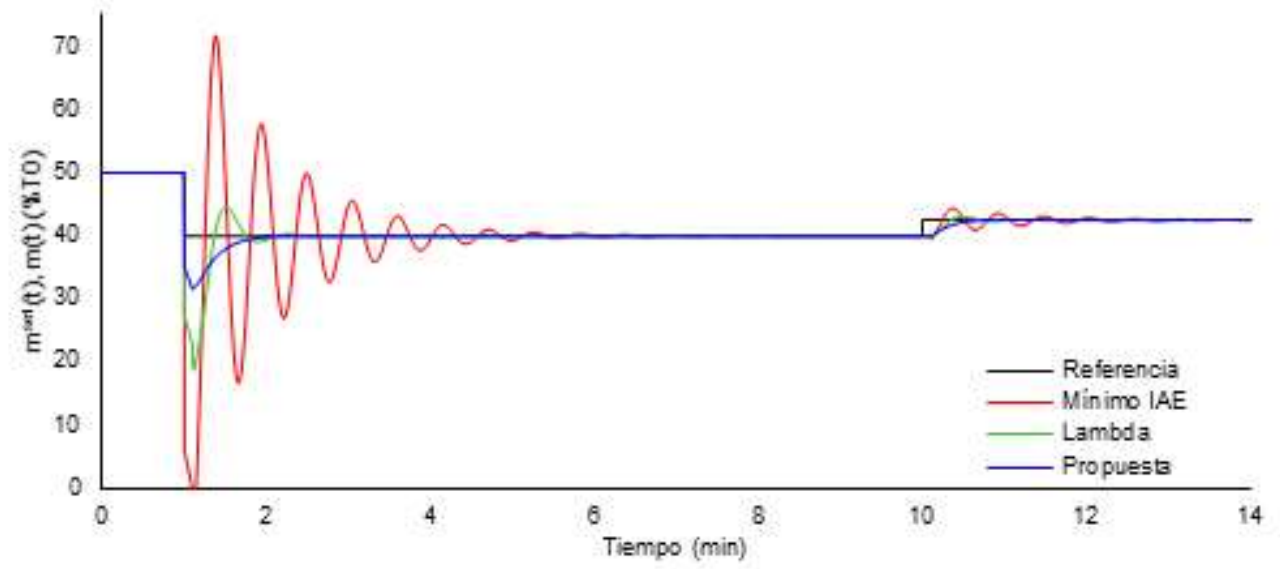

Fig. 9. Respuesta de la variable controlada $(C(t))$ para cada uno de los sets de sintonía, escenario de evaluación $2: K_{2}^{\prime}=0.5, \tau=1.5$ y $t_{0} / \tau=0.5$.

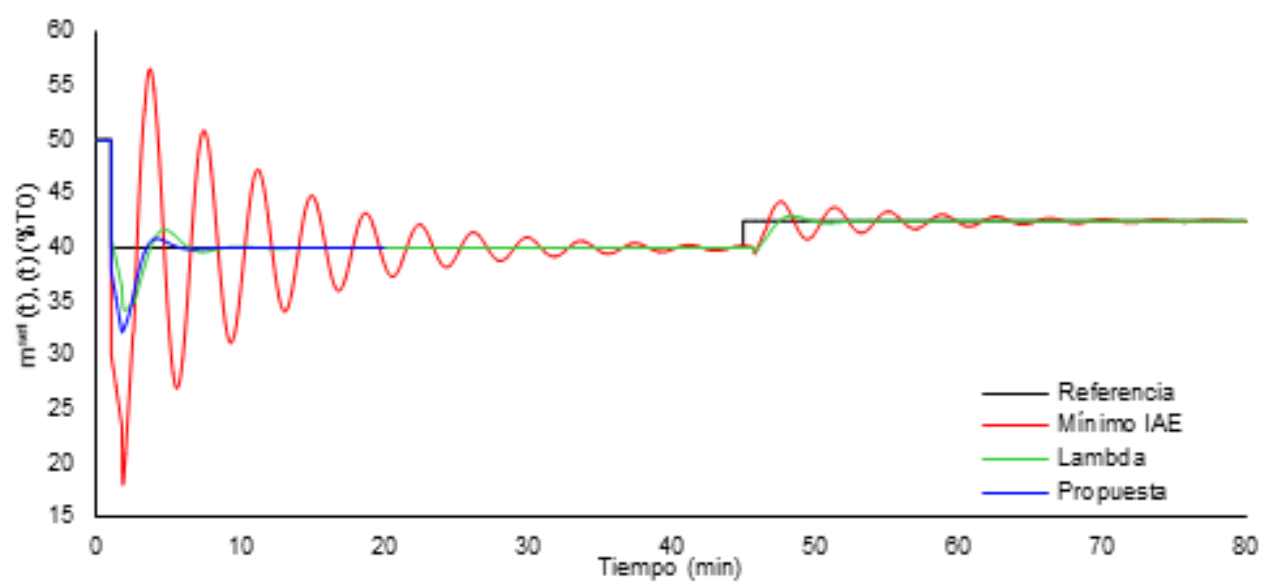

Fig. 10. Respuesta del controlador $(m(t))$ para cada uno de los sets de sintonía, escenario de evaluación $2: K_{2}^{\prime}=0.5, \tau=1.5$ y $t_{0} / \tau=0.5$.

Tabla 7. Parámetros cuantitativos de desempeño Integral absoluta del error (IAE) la varianza de la señal del transmisor y salida del controlador, para el escenario de evaluación 2.

\begin{tabular}{|c|c|c|c|c|}
\hline Ecuaciones de Sintonía & $I A E$ & $\sigma^{2}(C(t))$ & $\sigma^{2}(m(t))$ & $V o$ \\
\hline Mínimo IAE & 30,590 & 0,714 & 13,856 & 45,160 \\
\hline$\lambda^{\prime}$ & 11,240 & 0,423 & 0,637 & 12,300 \\
\hline Propuestas & 9,368 & 0,378 & 0,836 & 10,582 \\
\hline
\end{tabular}

El desempeño de las ecuaciones de sintonía desarrolladas fue evaluado en el modelo del fotobiorreactor ante cambios de setpoint (referencia) y perturbaciones para dos condiciones de operación particularmente difíciles de controlar. El desempeño de estas ecuaciones fue, además, comparado con el desempeño de las ecuaciones de sintonía $\lambda^{\prime}$ y mínimo IAE. La comparación fue hecha con criterios cualitativos, i.e. tiempo de estabilización y oscilaciones, y criterios cuantitativos, i.e. IAE y las varianzas de las señales del transmisor y el controlador respectivamente. Luego de la evaluación de desempeño y comparación con las ecuaciones $\lambda^{\prime}$ y mínimo IAE, las ecuaciones propuestas mostraron un mejor desempeño de acuerdo a los criterios cualitativos y cuantitativos establecidos.

\section{AGRADECIMIENTOS}

Este artículo es resultado del proyecto de investigación Implementación de proyectos de I+D (componentes Microalgas, Extractos de plantas y cadena productiva de la sábila, Producción 1,2 Pro-panodiol y Plataforma Informática) para promover el desarrollo y la transferencia tecnológica de cadenas productivas agroindustriales y la implementación de tecnologías de última gene-ración para el procesamiento de biocombustibles en el Departamento del Atlántico, el cual es financiado por el Departamento del Atlántico, a través de recursos del Sistema General de Regalías - Fondo de Ciencia, Tecnología e Innovación. 


\section{NOTACIÓN}

$\begin{array}{lll}\text { Símbolo } & \text { Significado } & \text { Unidades } \\ h & \text { Altura } & \mathrm{m} \\ C_{p} & \text { Calor específico } & \mathrm{kJ} / \mathrm{kg} \cdot \mathrm{K} \\ \mu & \text { Constante de proporcionalidad } & - \\ \rho & \text { Densidad } & \mathrm{kg} / \mathrm{m}^{3} \\ E & \text { Energía } & \mathrm{kJ} \\ \dot{q} & \text { Flujo de calor } & \mathrm{kJ} / \mathrm{s} \\ \dot{m} & \text { Flujo másico } & \mathrm{kJ} / \mathrm{s} \\ m & \text { Masa } & \mathrm{kg} \\ T & \text { Temperatura } & { }^{\circ} \mathrm{C} \\ \Gamma & \text { Variable de desviación } & - \\ V & \text { Volumen } & \mathrm{m}^{3} \\ \text { Subíndice } & \text { Significado } & \\ \text { ag } & \text { Refrigerante (Agua) } & \\ \text { amb } & \text { Ambiente circundante } & \\ i & \text { Condición final } & \\ 0 & \text { Condición inicial } & \\ \text { ss } & \text { Estado estable } & \\ \text { luz } & \text { Fuentes lumínicas } & \\ \text { air } & \left.\text { Intercambio de gases (Aire y } \mathrm{CO}_{2}\right) & \\ \text { med } & \text { Medio de cultivo } & \\ \text { al } & \text { Metabolismo microalgas } & \\ \text { reac } & \text { Reactor } & \end{array}$

\section{REFERENCIAS}

Barbosa, M., Janssen, M., Ham, N., Tramper, J., Wijffels, R. "Microalgae cultivation in air-lift reactors: Modeling biomass yield and growth rate as a function of mixing frequency". Biotechnology and Bioengineering, 82(2), pp.170-179 (2003)

Contreras, C., Peña, J., Flores, L., Cañizares, R. "Avances en el diseño conceptual de fotobiorreactores para el cultivo de microalgas". Interciencia, 28, pp. 450-456 (2003)

Janssen, M., Tramper, J., Mur, L., Wijffels, R. "Enclosed outdoor photobioreactors: light regime, photosynthetic efficiency, scale-up, and future prospects". Biotechnol Bioeng, 193-210 (2003)

Kiang, N., Siefert, J., Govindjee, Blankenship R. "Spectral Signatures of Photosynthesis. I. Review of Earth Organisms". Astrobiology, 7(1), pp. 222-251 (2007)

Mata, T., Martins, A., Caetano, N. "Microalgae for biodiesel production and other applications: A review". Renewable and Sustainable Energy Reviews, 14 (1), pp. 217-232 (2010)

Pramparo, L., Pruvost, J., Stuber, F., Font, J., Fortuny, A., Fabregat, A., Legentilhomme, P., Legrand, J., Bengoa, C. "Mixing and hydrodynamics investigation using CFD in a square-sectioned torus reactor in batch and continuous regimes". Chemical Engineering Journal, 137, pp. 386-395 (2008)

Richmond, A. "Handbook of Microalgal Culture: Biotechnology and Applied Phycology", Blackwell Science, Australia (2004)

Rosello Sastre, R., Csögör, Z., Perner-Nochta, I., Fleck-Schneider, P., \& Posten, C. "Scale-down of microalgae cultivations in tubular photo-bioreactors-A conceptual approach". Advances in Biochemical Engineering Science, 132(2), pp.127-133 (2007)

Sanchez, J., Cerón, M., García, F., Molina, E., Chisti, Y. "Growth and biochemical characterization of microalgal biomass produced in bubble column and airlift photobioreactors: studies in fed-bach culture". Enzyme and Microbial Technology, 31, pp. 1015-1023 (2002)

Sánchez, J., Fernández, J., Acién, F., Rueda,A., Pérez, J., Molina, E. "Influence of culture conditions on the productivity and lutein content of the new strain Scenedesmus almeriensis". Process Biochemistry, 43, pp. 398405 (2008)

Sierra, E., Acién, F. G., Fernández, J. M., García J. M., González, C., Molina, E. "Characterization of a flat photobioreactor for the production of microalgae". Chemical Engineering Journal, 138, pp. 136-147 (2008)

MathWorks, Optimization Toolbox TM User's Guide (2015),

http://www.mathworks.com/help/pdf_doc/optim/optim_tb.pdf. Acceso: 23 de Enero (2016)

Smith C. y Corripio A., Tuning of Feedback Controllers, Principles and Practices of Automatic Process Control, 3를 Edición, Editorial Wiley, 303-355, Hoboken, New Jersey, USA (2006) 